\title{
LIFT AND DRAG FORCE CALCULATION METHODS USING NON-LINEAR SECTION DATA. HISTORY AND RECENT RESEARCH
}

\section{E. Pakalnis}

\author{
VGTU Antanas Gustaitis Aviation Institute, Lithuania.E-mail: egidijus.pakalnis@tetrapak.com \\ Received 0707 2004, accepted 26072004
}

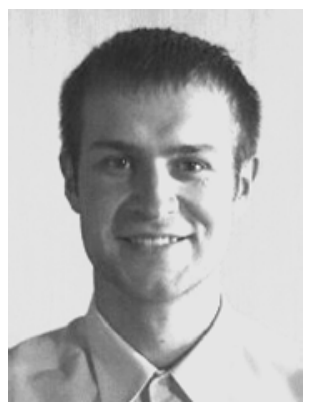

Egidijus PAKALNIS was born in 1974, in Biržai. In 1996 graduated Aviation Institute of Vilnius Gediminas Technical University. In 1998 received master's degree of aviation mechanics. From 1997 to 2002 worked in Antanas Gustaitis Aviation Institute as chief engineer. At present doctoral student of Vilnius Gediminas Technical University. Interest of research - low speed nonlinear aerodynamics. Author and co-author of seven scientific articles.

\begin{abstract}
In this article history and development of calculation methods of wing characteristics are presented. The development process includes the beginning in 1918 by Prandtl's classical lifting line theory and leads to resent research. The most attention is paid to non-linear section data implementation methods in the calculation of finite span wing. The research of Philips and Snyder, Barnes, and Sivells and Neely is discussed.
\end{abstract}

Keywords: lift, drag force, calculation methods of wing characteristics.

\section{First step - 1918 Prandtl's classical lifting line theory}

Developed during the period from 1911-1918, Prandtl's classical lifting line theory is the base of most methods used for calculations of change of lifting force of 4flat, low sweep angle, and moderate and high aspect ratio wings [18]. Prandtl's lifting line theory (LLT) uses a single unswept lifting line (or bound vortex) to model the circulation on the wing. The strength of this bound vortex, $\Gamma$, varies along the span. At any given span location, the change in $\Gamma$ is shown as trailing vorticity, which in turn causes induced velocities along the lifting line. LLT enables the computation of the $\Gamma$ distribution for which the accompanying induced velocities and the resulting effective angles of attack along the span support the $\Gamma$ distribution. For this purpose, the 1 Prandtl's LLT assumes a linear lift-curve slope for the airfoil sections that form the wing. This lift-curve slope is typically close to $2 \pi$ per radian.

As mentioned before, LLT is based on a linear relationship between section lift and section angle of attack. With this linear assumption, and with the assumption of a straight lifting line, the theory provides an analytical solution for the spanwise distribution of lift and induced drag acting on a finite lifting surface. The solution is in the form of an infinite sine series for the circulation distribution. Typically, the series is truncated to a finite series, and the coefficients in the finite series are evaluated by requiring the lifting-line equation to be satisfied at a number of spanwise locations equal to the number of terms in the series. Glauert first presented a very straightforward method [7]. The most popular method, based on Gaussian quadrate, was originally presented by Multhopp [14]. Most recently Rasmussen and Smith have presented a more rapidly converging method, based on a Fourier series expansion similar to that first used by Karamcheti [19, 9].

\section{Implementation of non-linear effects}

Tani developed the first successful technique in 1934 for handling nonlinear section lift-curve slopes in the LLT formulation [23]. In his technique, a spanwise bound vorticity $(\Gamma)$ distribution is first assumed. This distribution is used to compute the distribution of induced velocities and hence induced angles and effective angles of attack along the lifting line. The distribution of effective angles of attack is then used to find the operating $\mathrm{C}_{1}$ of the local section using known nonlinear $\mathrm{C}_{1}-\alpha$ data for the airfoil. A new $\Gamma$ distribution is then computed from the spanwise $C_{1}$ distribution. The iteration is carried out until $\Gamma$ distribution converges. This method was made popular by the NACA report of Sivells and Neely in 1947 that provides a detailed description of the method for unswept wings with arbitrary plan form and airfoil lift-curve slopes [22]. They apply this method for analysis of wings up to stall, i.e., until a wing angle of attack at any section on the wing has $C_{1}$ equal to $C_{l m a x}$.

Sivells and Neely presented a method for calculating wing characteristics by lifting-line theory using nonlinear 
section lift data [22]. Multhopp's system of multipliers isemployed to obtain the induced angle of attack directly from the spanwise lift distribution:

$$
\alpha_{i}=\frac{180}{4 \pi \sin \theta} \sum n A_{n} \sin n \theta
$$

Here the coefficients $A_{n}$ are determined from the known lift distribution points.

Also, the spanwise lift distribution is expressed as the trigonometric series:

$$
\frac{c_{l} \cdot c}{b}=\sum A_{n} \sin n \theta,
$$

Here $\quad c_{1}$ is the section lift coefficient, $\mathrm{c}$ is the section chord, $\mathrm{b}$ is the wingspan,

$A_{n}$ are the coefficients in the trigonometric series

$\cos \theta=2 y / b$, where $y$ is the section spanwise coordinate.

C. Sivells and Neely are subject to the limitations of the lifting-line theory upon which the methods are based. According to Mukherjee, Gopalarathnam, and Kim, at higher angles of attack, this approximation approach appears to fail [13].

According to Sears, Von Kerman noticed that Prandtl's lifting-line equation has no unique solutions for cases when the lift-curve slope becomes negative [21]. These non- unique solutions include both symmetrical and antisymmetric lift distributions even when the geometry and onset flow are both symmetric.

The investigation suggested by Von Karman was carried out and was reported in a 1939 thesis by Schairer working under the supervision of Sears [20]. Sears presents some of Schairer's results for a flat, untwisted elliptic wing of aspect ratio 10,19 operating beyond stall. The results show solutions consisting of asymmetric lift distributions (in addition to a classical symmetric solution) with large associated rolling moments for a narrow range of angles of attack just beyond stall.

\section{Numerical solutions of LLT in comparison with panel and CFD methods}

Numerical solutions of Prandtl's lifting line theory were also developed and are still in use. The most wellknown works, that use these methods are the works of McCormick and Anderson [12, 1]. Phillips and Snyder present some information about their work [16].

McCormick has presented a numerical method that can be used for a single lifting surface having a straight lifting line [12]. This method is based on applying the two-dimensional Kutta-Joukowski law to the 3D flow and neglects the downwash generated by the bound vorticity. Results obtained from this method are essentially identical to those obtained from the series solution. A numerical lifting-line method that relaxes the assumption of linearity between section lift and section angle of attack has also been developed by Anderson [1]. For a single straight lifting surface, this method gives good agreement with experimental data at angles of attack both below and above stall. However, the method still assumes a straight lifting line and ignores the downwash produced by the bound vorticity. Thus, this method only applies to a single lifting surface without sweep and no dihedral.

Unlike the classical lifting-line solution, the method presented by Phillips and Snyder is not based on a linear relationship between section lift and section angle of attack [16].

Phillips and Snyder used the numerical lifting line method for a wing model [11]. For a comparison with results obtained from panel methods Phillips and Snyder used PMARC [10, 2]. This code was developed by NASA Ames Research Center and is one of the most efficient numerical panel codes available. PMARC uses a flat quadrilateral panel with uniform source and doublet distributions on each panel. This code also accounts for the effects of wake rollup, using an unsteady wake development approach.

For an inviscid CFD comparison, the commercial code WIND was chosen [5]. This code uses a nodecentered finite volume approach to solve Euler equations on a structured grid.

The lift coefficient predicted by all three methods was in agreement with experimental observations for tested wings. It should also be noticed that for a straight wing, the induced drag predicted by both the numerical lifting-line method and by PMARC was in agreement with experimental data, whereas WIND gave an induced drag that is somewhat higher.

For a swept wing (45 degrees) none of the tested methods could predict the induced drag with the desired accuracy. The induced drag predicted by PMARC was about $40 \%$ less than that observed experimentally. Both the numerical lifting-line method and the CFD solution gave induced drag values that were about 25 percent above the experimental values.

The over-prediction of induced drag by the CFD code for both a straight wing and a swept wing is likely due to numerical viscosity and is expected.

The reason that the numerical lifting-line method over- predicts induced drag for the swept wing, but not for a straight wing, is in the aerodynamic center of the swept wing. The foundation of lifting-line theory requires the bound vorticity to follow the chord wise aerodynamic center of a wing. Usually the assumption is made that lifting-line follows the wing quarter chord, what presents a theoretical aerodynamic center according to a theory of thin airfoil. In reality, the experimental results of Weber and Brebner show that, near the spanwise midpoint of a highly swept wing, the aerodynamic center moves considerably after of the wing quarter chord (also the lifting line is curved forward near the wing tips) [26]. Barnes presents a correction technique for the aerodynamic center shift of swept and tapered wings [3]. Still there is no simple means to predict the true aerodynamic center of a highly swept wing in the region near the spanwise midpoint. 
Taking into account that the lifting line method requires a known relationship between the section lift coefficient and angle of attack, the method of Phillips and Snyder predicts stall by using a semi-empirical correction to the potential flow solution [16]. For that reason, the authors conclude that their method should be used with extreme caution for angles of attack beyond stall.

\section{Vortex step method and other modified lifting-line methods}

The works of Mutteperl and Weisinger made a base for the so-called Finite-Step method or Vortex Step Method, which was also developed from Prandtl's lifting line theory as a numerical solution [15, 27]. It differs from Prandtl's theory by the location of control points at the three-quarter chord, instead of the one quarter. This condition allowed taking into account the sweep of a calculated wing. Later, Campbell and Blackwell simplified their method $[6,4]$.

The simplification involves replacing the continuous lifting line of varying strength by a discrete system of horseshoe vortices, each of which is of constant strength. The resulting method allows one to couple sectional (twodimensional) viscous results with inviscid wing (threedimensional) theory in order to determine the total aerodynamic coefficients for configurations including wings with dihedrals, endplates/winglets, pylons, and for biplanes, joined wings, etc. [3]. The present method has advantages over traditional panel methods because this method is significantly faster than traditional panel methods, and it also incorporates the critical viscous nature of high-lift devices.

Although the modified Weisinger method reduces the lifting surfaces to flat plates, various airfoil shapes, including high-lift configurations, can be successfully modeled.

Van Dam, Vander Kam, and Paris, in order to compute the load distribution in the subsonic compressible flow of arbitrary wings and lifting surface arrangements, used a modified lifting-line method, mentioned above [25]. They conclude that their method provides the necessary accuracy to be useful at the conceptual and preliminary design stages, while being fast enough to be used during the early stages of design where thousands of iterations can be examined.

Barnes presents the most resent research that reflects the Vortex Step Method [3]. In his research Barnes shows that the Vortex Step Method can be more accurate than its CFD alternative when predicting lift slope. He presents an EVSM (semi-Empirical Vortex Step Method), which includes empirical adjustments in lifting line position and shape.

The most important semi-empirical adjustment implemented by EVSM represents a lifting line shift to obtain a more accurate aerodynamic center position. Four empirical adjustments are made in EVSM presented by Barnes:

1. Adjustment of the aerodynamic center of an unswept wing as well as correction for the aerodynamic center of swept and tapered wings;
2. Adjustment that relates to the shape of the lifting line at the wing root and tip. Although this adjustment has only a minor effect on the calculated lift slope, it offers some improvement in the accuracy of the calculated lift loading.

3. Adjustment that addresses the optimistic lift prediction of the Vortex Step Method for swept, constant-chord wings. This adjustment reduces the lift slope by shifting the downwash line forward.

4. Adjustment that take into account the loss in flap effectiveness due to sweep and finite span fraction.

Piszkin and Levinsky developed a nonlinear liftingline method based in part on the iterative method originally conceived by Tani $[17,23]$. Their model uses a single chord wise row of horseshoe vortices distributed along the span, with the bound vortex aligned with the local quarter-chord line. The boundary condition of zero normal flow is applied at the control point, which is the three-quarter-chord location for each horseshoe vortex. As a consequence of using a single chord wise horseshoe vortex, the method is restricted to wings of moderate to high aspect ratio. It must be mentioned that this method differs from Prandtl's classical LLT in the implementation of the boundary condition.

In order to account for the nonlinear lift-curve slopes, Piszkin and Levinsky use the iterative technique described earlier. At each step of the iteration, the downwash computed using $\Gamma$ distribution from the previous time step is used to compute the change in the $\Gamma$ distribution using the airfoil lift curve. This change, multiplied by a specified damping factor, $\mathrm{C}$, is then added to the old $\Gamma$ distribution to obtain the new $\Gamma$ distribution for the next iteration. A damping factor of $\mathrm{C}<1$ is required to stabilize the iterations, although it results in a larger number of iterations for convergence. Unlike in the traditional LLT, where the effective section angle of attack distribution is computed as part of the solution, here the effective section angle of attack distribution is not readily available.

Using their method, Piszkin and Levinsky found that multiple converged solutions are possible, including some that have saw-tooth type oscillations in the spanwise lift distributions (also observed in 13). To avoid these oscillations, they used a switching logic that restarts the iteration procedure with an initial distribution having a zero induced $\alpha$ for any wing section found to be stalled.

An entirely different approach to the use of nonlinear section data was developed by Tseng and Lan [24]. While their main focus was on vortex-dominated flows on low aspect ratio fighter-type wings at high $\alpha$, they incorporated the effect of boundary-layer separation by iteratively reducing the angle of attack at each section of the wing. The reduction at any given wing section is determined by the difference between the potential flow solution and the viscous $C_{1}$ from the nonlinear section $C_{1^{-}}$ $\alpha$ curve.

In all methods that use nonlinear section data, the main objective is that, for the final solution of the threedimensional flow, the $\Gamma$ distribution across the span is consistent with the distribution of the effective $\alpha$ for each 
section and that the $C_{1}$ and $C_{m}$ for each section is consistent with the effective $\alpha$ for that section and the section $\mathrm{C}_{1}-\alpha$ and $\mathrm{C}_{\mathrm{m}}-\alpha$ data. Mukherjee, Gopalarathnam, and Kim achieve that condition by finding the effective reduction in the camber distribution for each section along the span [13]. For the iteration process, a multidimensional Newton iteration is used that automatically takes into account the effect of the decambering at one section on the lift at all of the other sections. The authors conclude that experimental data is needed to validate the accuracy of their method and to determine its application range.

Another possibility to take into account non-linear section data in calculation of wing characteristics is presented in the research of Jacob [8]. His method combines an inviscid 3d-lifting surface theory with a $2 \mathrm{~d}$ airfoil theory that includes boundary layer calculations and a displacement model for rear separation. The total iterative procedure allows the complete wing characteristics including maximum lift and post stall to be predicted but is restricted to wings with moderate to high aspect ratio and low sweep. For taking account of the finite span of the wing, Jacob uses the linear lifting surface theory in combination with section data of viscous flow.

The following steps present the procedure for nonlinear section data evaluation used by Jacobs:

1. Induced flow angles for each wing section are computed with 3-d lifting surface theory. Effective angle of attack is determined in that way:

$$
\alpha_{e}=\alpha_{g}-\Delta \alpha
$$

Here:

$$
\begin{aligned}
& \Delta \alpha=F \cdot \Delta \alpha^{* *}+(1-F) \Delta \alpha^{*} ; \mathrm{F}=0,33 \\
& \Delta \alpha^{*}=\alpha^{*}-\left(\alpha^{*}\right)_{2-d} \\
& \Delta \alpha^{* *}=\alpha^{* *}-\left(\alpha^{* *}\right)_{2-d}
\end{aligned}
$$

Where induced angles of attack of the 3-d flow $\alpha^{*}$ and $\alpha^{* *}$ are calculated from the system of linear equations based on the 3-d lifting surface theory. 2-d angles of attack are calculated in the following manner:

$$
\begin{aligned}
& \left(\alpha^{*}\right)_{2-d}=\left(C_{l}-C_{m}\right) / 2 \pi ; \\
& \left(\alpha^{* *}\right)_{2-d}=\left(C_{l}+8 C_{m}\right) / 2 \pi
\end{aligned}
$$

2. Section $\mathrm{Cl}$ is computed from the 2-d airfoil theory for $\alpha_{e}$.

3. From $\mathrm{Cl}$ and the section chord new lift distribution is obtained.

4. Procedure is repeated.

A condition of the iteration procedure used by Jacob is that the iteration procedure is stopped, if

$$
\left|\gamma^{(j)}-\gamma^{(j+1)}\right| / \operatorname{Max} \gamma \mid \leq \varepsilon
$$

where: $\gamma=C_{l} \cdot c / 2 s$, here $\mathrm{c}$ is the chord of a wing section, and

$\mathrm{s}$ is the wing span.

The Maximum number of iterations is limited to 50 and accuracy is set to $\varepsilon=0,0003$, which usually takes from 20 to 40 iterations.

\section{Conclusions}

Prandtl's lifting line theory has developed into various methods of calculation for a finite wing during the last century. The references presented here prove, that the model presented by Prandtl, with some alterations and the use of a modern computer, can be used to predict inviscid forces acting on a finite wing of high aspect ratio with an accuracy compatible to a modern panel methods and CFD. Great interest in the theory shows that it is still in use at present time.

\section{References}

1. Anderson J.D., Corda S., Van Wie D.M. Numerical Lifting-Line Theory Applied to Drooped LeadingEdge Wings Below and Above Stall // Journal of Aircraft. - 1980. - Vol 17, No 12. - P. 898 - 904.

2. Ashby D.L., Dudley M.R., Iguchi S.K. Development and Validation of an Advanced Low-Order Panel Method, NASA TN-101024. - 1988.

3. Barnes J.P. Semi-Empirical Vortex Step Method for the lift and induced drag loading of 2D and 3D Wings, Society of Automotive Engineers/ SAE. - 1997 Oct. Paper 975559.

4. Blackwell J.A. A Finite-Step Method for Calculation of Theoretical Load Distributions for Arbitrary Lifting-Surface Arrangements at Subsonic Speeds. NASA TN D-5335. - 1969 July.

5. Bush R.H., Power G.D., Towne C.E. WIND: The Production Flow Solver of the NPARC Alliance/ AIAA. - 1998. - Paper 98-0935.

6. Campbell G.S. A Finite-Step Method for the Calculation of Span Loadings of Unusual Plan Forms. NACA RM L50L13. - 1951.

7. Glauert H. The Elements of Aerofoil and Airscrew Theory / $2^{\text {nd }}$ ed. - Cambridge: Cambridge Univ. Press, 1959. - P. 142 - 145.

8. Jacob K. Computation of the Flow around Wings with Rear Separation // Journal of Aircraft. - 1984. Vol 21. - P. 97 - 98.

9. Karamcheti K. Elements of Finite Wing Theory, Ideal-Fluid Aerodynamics. - Wiley, New York, 1966. - P. 535 - 567.

10. Katz J., Maskew B. Unsteady Low-Speed Aerodynamics Model for Complete Aircraft Configurations // Journal of Aircraft. - 1988. - Vol 25, No 4. - P. 302 - 310.

11. Katz J., Plotkin A. Lifting-line solution by Horseshoe Elements, Low-Speed Aerodynamics, from wing theory to Panel methods. - McGraw-Hill, New York, 1991. - P. 379 - 386.

12. McCormick B.W. The lifting-Line Model, Aerodynamics, Aeronautics and Flight Mechanics / $2^{\text {nd }}$ ed. - Wiley, New York, 1995. - P. 112 - 119. 
13. Mukherjee R., Gopalarathnam A., Kim S.W. An Iterative decambering Approach for Post-Stall prediction of Wing Characteristics Using Known Section Data. $41^{\text {st }}$ AIAA Aerospace Sciences Meeting, January 6-9, 2003/Reno, Nevada / AIAA. - 2003. Paper 1097.

14. Multhopp H. Method for Calculating the Lift Distribution of Wings, ARC Rep. Mem. 2884. - 1955.

15. Mutteperl W. The Calculation of Span Load Distributions on Swept-Back Wings. NACA TN 834. - 1941 Dec

16. Phillips W.F., Snyder D.O. Modern Adaptation of Prandtl's Classic Lifting-Line Theory // Journal of Aircraft. - 2000. - Vol 37, No 4. - P. 662 - 670.

17. Piszkin S.T., Levinsky E.S. Nonlinear Lifting Line Theory for Predicting Stalling Instabilities on Wings of Moderate Aspect Ratio, Tech. Rep. / General Dynamics Convair Report CASD-NSC-76-001. 1976 June.

18. Prandtl L. Application of Modern Hydrodynamics to Aeronautics. NACA 116. - 1921 June.

19. Rasmussen M.L., Smith D.E. Lifting-line Theory for Arbitrary Shaped Wings // Journal of Aircraft. - 1999. - Vol 22, No 2. - P. 340 -348.

20. Schairer R.S. Unsymmetrical Lift Distributions on a Stalled Monoplane Wing: Master's thesis / California Institute of Technology. - 1939.
21.Sears W.R. Some Recent Developments in Airfoil Theory // Journal of the Aeronautical Sciences. - 1956 May. - Vol. 23. - P. 490 - 499.

22. Sivells J.C., Neely R.H. Method for Calculating Wing Characteristics by Lifting-Line Theory Using Nonlinear Section Lift Data / NACA TN. - 1947 April. - No 1269.

23. Tani I. A Simple Method of Calculating the Induced Velocity of a Monoplane Wing, Rep. - Tokyo: Aero. Res. Inst. Tokyo Imperial University, 1934 August. Vol 9,3, No 111.

24. Tseng J.B., Lan C.E. Calculation of Aerodynamic Characteristics of Airplane Configurations at High Angles of Attack. NASA CR 4182. - 1988.

25. Van Dam C.P., Vander Kam J.C., Paris J.K. Design-Oriented High-Lift Methodology for General Aviation and Civil Transport Aircraft // Journal of Aircraft. - 2001. - Vol 38, No 6. - P. 1076 - 1084.

26. Weber J., Brebner G.G. Low-Speed Tests on 45-deg Swept Back Wings, Part I: Pressure Measurements on Wings of Aspect Ratio 5, Report and Memoranda 2882. - London: Aeronautical Research Council, 1958.

27. Weisinger J. The lift distribution of Swept-Back wings /NACA TM 1120. - 1947 March. 